\title{
Magnetic Resonance Imaging Evaluation of the Painful Total Knee Arthroplasty
}

\author{
Darryl B. Sneag, MD ${ }^{1,2}$ Eric A. Bogner, MD ${ }^{1,2}$ Hollis G. Potter, MD ${ }^{1,2}$ \\ ${ }^{1}$ Department of Radiology and Imaging, Hospital for Special Surgery, \\ New York, New York \\ 2 Department of Radiology, Weill Medical College of Cornell University, \\ New York, New York \\ Address for correspondence Darryl B. Sneag, MD, Department of \\ Radiology and Imaging, Hospital for Special Surgery, 535 East 70th \\ Street, New York, NY 10021 (e-mail: sneagd@hss.edu). \\ Semin Musculoskelet Radiol 2015;19:40-48.

\begin{abstract}
Keywords

- total knee arthroplasty

- MRA

- complications

Optimization of metal artifact reduction pulse sequences over the past decade has rendered MRI valuable in knee arthroplasty assessment. MRI can reliably predict the presence and extent of infection, component loosening and polyethylene wear, and component malrotation, and it can evaluate the integrity of surrounding soft tissue structures. Using dynamic contrast-enhanced angiographic techniques, vascular pathology such as pseudoaneurysm formation and recurrent hemarthrosis can also be assessed.
\end{abstract}

Total knee arthroplasty (TKA) is an increasingly common surgical procedure to improve function and alleviate pain in patients with symptomatic advanced knee osteoarthritis. In 2012, > 670,000 procedures were performed in the United States, more than double the number performed a decade prior, in patients with a mean age of 66 years. ${ }^{1}$ Factors contributing to this increase include population growth, with the greatest increase in TKAs performed in individuals 45 to 64 years of age; expanded indications for TKA particularly in younger individuals ( $<65$ years old); obesity; decreased postoperative complications, particularly infection; and a greater patient demand possibly fueled by direct consumer advertising. ${ }^{2}$ Despite generally successful outcomes and long implant survival, the rise in primary knee replacements has resulted in an increased number of revision procedures. $^{3}$

Imaging is the cornerstone in the diagnostic algorithm of the painful knee arthroplasty. Radiography should be the initial modality obtained because it is readily available, the least expensive, and can sometimes quickly reveal the cause of pain, such as a fracture or conspicuous component loosening. Radiographs, however, lack sensitivity and specificity for the degree of osteolysis ${ }^{4,5}$ and are extremely limited in evaluating synovitis type and extent, the integrity and position of polyethylene components, and the presence of infection. Computed tomography (CT) provides greater definition of bone stock, the degree of osteolysis, and extension of periprosthetic fracture. Optimizing acquisition and particularly postprocessing parameters, such as using iterative reconstruction, can substantially reduce beam hardening artifact that would otherwise impede implant assessment. ${ }^{6}$ However, CT lacks the inherent soft tissue contrast necessary to evaluate the supporting ligamentous structures and synovium. CT arthrography allows for detection of joint debris including polyethylene fragments that may have fractured and dislodged (-Fig. 1). Ultrasound can be used to address specific clinical concerns (e.g., patellar clunk, tendon or ligament abnormalities, or recurrent hemarthrosis), provides dynamic assessment, and serves as an alternative to fluoroscopy in guiding joint aspiration and the occasional biopsy.

Historically, MRI has served a very limited role in evaluating patients following knee arthroplasty due to susceptibility artifact from metallic hardware. Over the past decade, however, modification of acquisition parameters as well as the more recent development of novel pulse sequences such as multiacquisition variable-resonance image combination (MAVRIC) and slice encoding for metal artifact correction (SEMAC) have successfully reduced susceptibility artifact so that high-resolution images with exceptional osseous and soft tissue contrast are attainable. ${ }^{7-9}$ At our institution, orthopedists routinely obtain knee MRI examinations either to answer specific clinical questions such as extent of
Issue Theme Imaging of Joint Replacements; Guest Editor, Theodore T. Miller, MD, FACR
Copyright $\odot 2015$ by Thieme Medical Publishers, Inc., 333 Seventh Avenue, New York, NY 10001, USA. Tel: +1(212) 584-4662. 

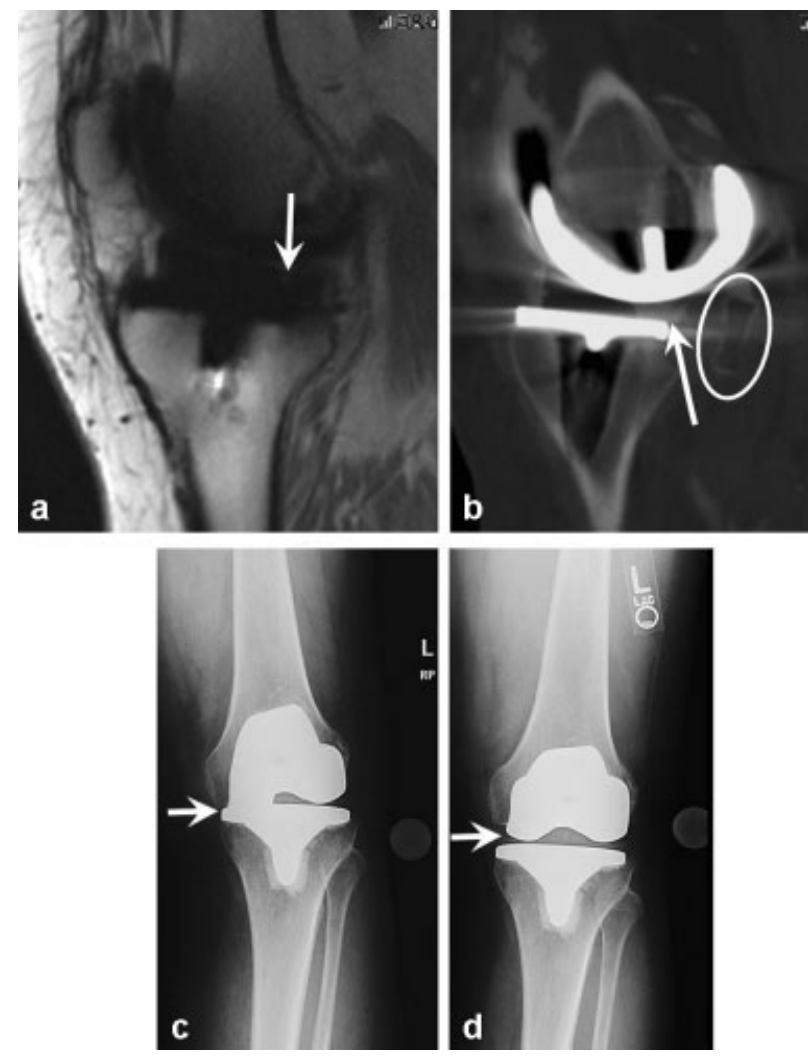

Fig. 1 A 77-year-old man status post left total knee arthroplasty 2 years prior with pain following a fall into a pothole 2 weeks ago. (a) Sagittal multiacquisition variable-resonance image combination fast spin-echo MR image demonstrates a minimally displaced fracture along the posteromedial aspect of the polyethylene tibial insert (arrow). (b) Sagittal reformatted image from a computed tomography (CT) arthrogram performed 2 months after the MRI confirms the fracture (arrow), which has progressed with a large fragment (oval) now displaced into a partially ruptured popliteal cyst filling with contrast via communication with the knee joint proper. (c) Frontal weightbearing knee radiograph at the time of CT compared with that from 2 years ago (d) demonstrates interval collapse of the medial joint space (arrows) reflecting the fractured polyethylene insert. polymeric wear-induced synovitis and integrity of the polyethylene component or to address the common complaint of nonspecific knee pain.

The most common reasons for TKA failure in decreasing frequency are aseptic loosening, infection, instability, periprosthetic fracture, and arthrofibrosis. ${ }^{10}$ For early revision ( $<2$ years from the primary surgery), infection was the most common reason for failure in the study of 781 revision TKAs by Sharkey et al. ${ }^{10}$ Interestingly, the incidence of polyethylene wear, with or without osteolysis, only accounted for 3.5\% of revision TKA procedures. Other less common but important mechanisms of failure include malrotation/malalignment and extensor mechanism deficiency.

\section{Complications}

\section{Osseous Integration, Aseptic Loosening, and Polyethylene Wear}

Adequate fixation of the knee arthroplasty into the host bone is necessary to ensure optimal function and implant survivorship. Knee implants can be fixed with or without cement, although in the United States, more commonly cement fixation is performed. ${ }^{11}$ Osseous integration can be limited by fibrous membrane formation along the bone-implant and bone-cement interfaces, in which a synovial-like or fibrous membrane is thought to form in response to mechanical stress and where synoviocytes may release proresorptive cytokines. ${ }^{12,13}$ Although the significance and effect of fibrous membrane formation on component loosening are yet to be determined, ${ }^{14}$ its theoretical potential to progress to aseptic loosening warrants mention of its presence in the imaging report. A fibrous membrane is assumed from the presence of a smooth intermediate- to high-signal region, $<2 \mathrm{~mm}$ in thickness and bordered by a hypointense line, at the interface of the host bone and cement or implant. ${ }^{12}$ It is our experience that fibrous membrane formation is most frequently detected at the patellar component cement interface (-Fig. 2). Bone resorption or osteolysis is identified by a layer $>2 \mathrm{~mm}$
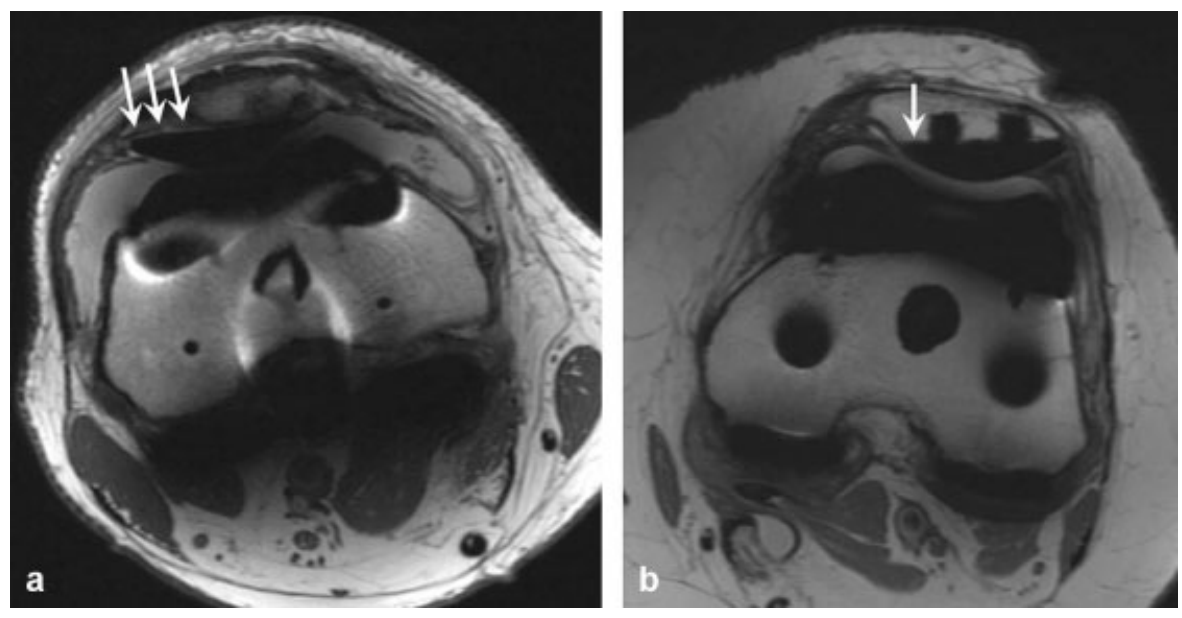

Fig. 2 Axial fast spin-echo MR images in two different patients. (a) Fibrous membrane is depicted as a thin layer of signal hyperintensity at the implant-bone interface of the patellar component (arrows). (b) Compare with the normal integration of the patellar component (arrow). 

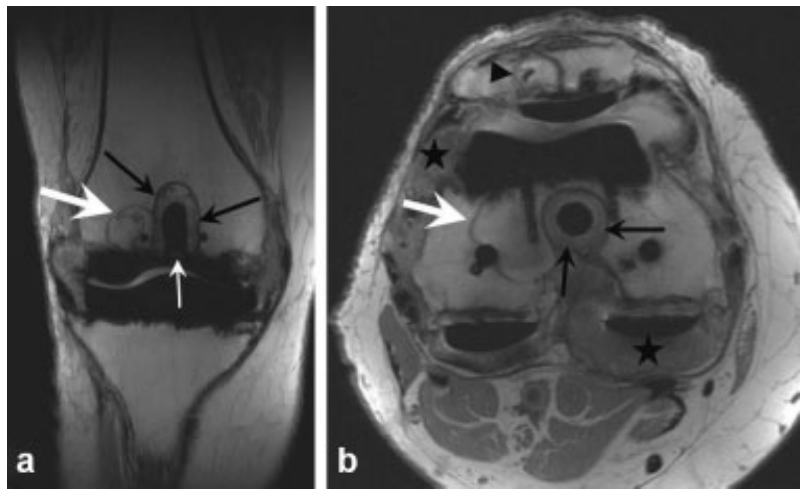

Fig. 3 (a) Coronal and (b) axial fast spin-echo MR images in a 48-yearold man status post right total knee arthroplasty 6 years ago with pain. There are large areas of osteolysis containing internal foci of polymeric debris along the lateral femoral flange (thick white arrows, $a$ and $b$ ) and patellar component (arrowhead, b). Bulky intermediate signal intensity synovial debris (stars, B), typical of polymeric wear, distends the posterior and lateral recesses. Also noted is circumferential osteolysis, indicating loosening, around the femoral post (thin black arrows, a and b), which has a hyperintense linear fracture at its base (thin white arrow, a).

between the bone-implant or bone-cement interface and may have irregular borders (-Fig. $\mathbf{3}$ ).

Osteolysis is a major complication of knee arthroplasty that can result in aseptic loosening and periprosthetic fracture and is a result of macrophage phagocytosis of particle debris, typically polyethylene (also known as polymeric debris), which results in osteoclast upregulation and osteo- blast downregulation. ${ }^{15}$ The ability to quantify the extent of osteolysis is important because larger lesions tend to progress more quickly over time compared with smaller ones. ${ }^{16}$ Although small areas of osteolysis may be monitored, the presence of circumferential areas of bone resorption around an implant suggests component loosening and may require revision surgery. It is also critical to provide an orthopedist planning revision surgery with an accurate road map of available bone stock. Both MRI and CT afford far superior sensitivity and specificity for the detection of osteolysis compared with radiographs. However, MRI may be more sensitive for detecting osteolysis around the curved flanges of the femoral component compared with $\mathrm{CT}$, and it does not expose a patient to ionizing radiation. ${ }^{4,5}$ It is important to note that femoral components composed of conventional cobalt/chrome/molybdenum alloy may result in significant susceptibility artifact and be more challenging to evaluate than those made of zirconium. ${ }^{17}$ The sagittal MAVRIC sequence, routinely obtained as part of our knee arthroplasty protocol ( - Table 1), allows for improved detection of osteolysis around the femoral flanges compared with metal artifact-reduction fast-spin echo (FSE) images, ${ }^{18}$ and it is also helpful in evaluating the interfaces along the tibial baseplate. Axial and coronal metal artifact-reduction FSE images are most useful for evaluating the interface around the patellar component and its pegs. Osteolysis is frequently identified around one or two cemented pegs of a patellar component, but unless osteolysis is detected around all three pegs, the patellar component is not considered loose per our experience.

Table 1 Sample protocol for MRI of knee arthroplasty

\begin{tabular}{|l|l|l|l|l|l|}
\hline Timing parameters & Axial FSE & Coronal FSE & Sagittal FSE & $\begin{array}{l}\text { MAVRIC } \\
\text { sagittal IR }\end{array}$ & $\begin{array}{l}\text { MAVRIC } \\
\text { sagittal FSE }\end{array}$ \\
\hline TR, ms & $4000-6000$ & $4000-6000$ & $4000-6000$ & $4000-6000$ & $4000-6000$ \\
\hline TE, ms & 28 & 28 & 30 & 17 & 8 \\
\hline Flip angle, degrees & 160 & 160 & 160 & 110 & 110 \\
\hline TI, ms & - & - & - & 150 & - \\
\hline ETL & 16 & 16 & 16 & 24 & 24 \\
\hline RBW, $\mathrm{kHz}$ & 125 & 125 & 125 & 125 & 125 \\
\hline FOV, $\mathrm{cm}$ & 21 & 21 & 20 & 21 & 22 \\
\hline Matrix & $512 \times 256$ & $512 \times 256$ & $512 \times 320$ & $256 \times 192$ & $512 \times 256$ \\
\hline Slice thickness, mm & 3.5 & 3.0 & 3.5 & 4 & 3 \\
\hline Interslice gap, mm & 0 & 0 & 0 & 0 & 0 \\
\hline NEX & 4 & 5 & 4 & 2 & 1.5 \\
\hline Tailored RF & Yes & Yes & Yes & - & - \\
\hline Variable BW & Yes & Yes & Yes & Yes & Yes \\
\hline Frequency direction & Anterior to & Right to left & Anterior to & $\begin{array}{l}\text { Anterior to } \\
\text { posterior }\end{array}$ & $\begin{array}{l}\text { Anterior to } \\
\text { posterior }\end{array}$ \\
\hline
\end{tabular}

Abbreviations: BW, bandwidth; ETL, echo train length; FOV, field of view; FSE, fast spin echo; IR, inversion recovery; MAVRIC, multiacquisition variableresonance image combination; NEX, number of excitations; RBW, receiver bandwidth; RF, radiofrequency; TE, echo time; TI, inversion time; TR, repetition time.

Note: The reported RBW is reported as half bandwidth. To convert to BW per pixel, use the following formula: $2^{*}$ (half bandwidth)/(readout matrix). 

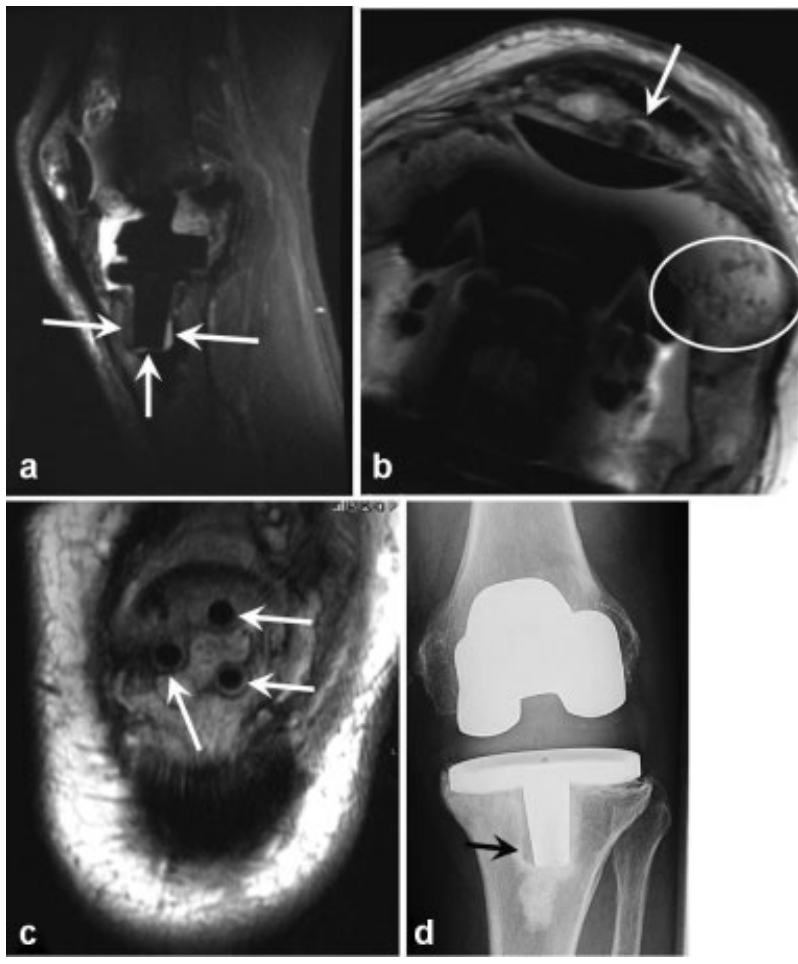

Fig. $4 \mathrm{MRI}$ of the left knee in a 55-year-old man status post left total knee arthroplasty 3 years ago. (a) Sagittal multiacquisition variable-resonance image combination inversion recovery and (b) axial fast spin-echo (FSE) MR images demonstrate a bulky synovitis. The axial FSE image clearly demonstrates the intermediate signal particulate appearance of the synovitis (oval), typical of polymeric wear. Circumferential signal hyperintensity surrounding the tibial (arrows, a) and patellar components (arrows, b and c, a coronal FSE image), including its pegs, strongly suggests loosening, and the tibial component has also shifted. (d) The frontal knee radiograph demonstrates radiolucency surrounding the tibial component (arrow) corresponding to the MR findings, but loosening of the patellar component cannot be appreciated despite Merchant and lateral projections (not shown here).

Multiple variables, including implant design and patient factors (e.g., weight and activity level) affect polymeric wear after TKA. ${ }^{19,20}$ Advancement in polyethylene design has led to the development of highly cross-linked polyethylene tibial and patellar components that boast lower wear rates compared with conventional ultra-high molecular weight polyethylene. $^{21}$ Despite this, polymeric wear remains an important cause of synovitis, osteolysis, and ultimate loosening. On MRI, polyethylene wear-induced synovitis is best recognized on moderate echo time sequences by thick particulate-appearing and sometimes bulky synovitis (-Fig. 3d) of low to intermediate signal intensity with a variable amount of fluid. ${ }^{12}$ "Particulate" refers to small pleomorphic, sometimes confluent foci that can be identified within the synovial distension (-Fig. 4).

\section{Infection}

The incidence of periprosthetic joint infection following TKA ranges from $0.4 \%$ to $2 \%{ }^{22}$ Although the definitive diagnosis of joint infection relies on aspiration of joint fluid and subsequent microbiological and histologic analysis, imaging can be predictive. Unless osseous destruction or periostitis is present, radiographs often do not reveal the presence of infection and cannot qualify the type of synovitis. Dual nuclear medicine radiotracer scans, most commonly leukocyte-bone marrow imaging with technetium $99 \mathrm{~m}$ sulfur colloid, can be used to detect infection around a TKA. However, results are inconsistent and interpretation complicated. ${ }^{23}$ On MRI, a "lamellated" or multiple-layered appearance of the synovium suggests infection (-Fig. 5). Using this finding as a criterion for infection, Plodkowski et al demonstrated sensitivity of 86 to $92 \%$ and specificity of 85 to $87 \%$ for accurately diagnosing an infected knee arthroplasty. $^{24}$

\section{Polyethylene Liner Complications}

In early knee replacement designs, the polyethylene-bearing surface was directly molded onto the tibial baseplate. To provide multiple sizing options, modern designs now use modular components that are locked into place at surgery. ${ }^{11}$ Although numerous polyethylene insert designs are available, the two major types are fixed bearing, in which the polyethylene insert is locked into the tibial tray, and mobile bearing, in which the polyethylene insert can rotate or translate over
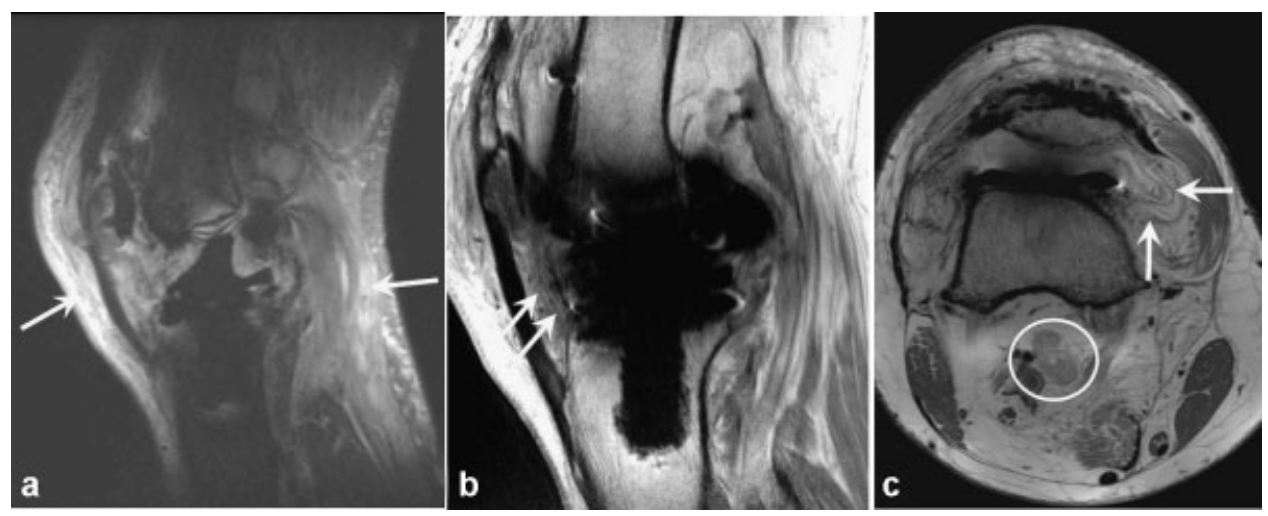

Fig. 5 (a) Sagittal multiacquisition variable-resonance image combination inversion recovery and (b) sagittal and (c) axial fast spin-echo MR images in a 77-year-old man 16 months status post left total knee arthroplasty demonstrate hypertrophic fluid-signal intensity synovitis with a lamellated appearance (arrows, b and c). Note the prominent extracapsular subcutaneous edema (arrows, a) and reactive popliteal lymphadenopathy (oval, c). 

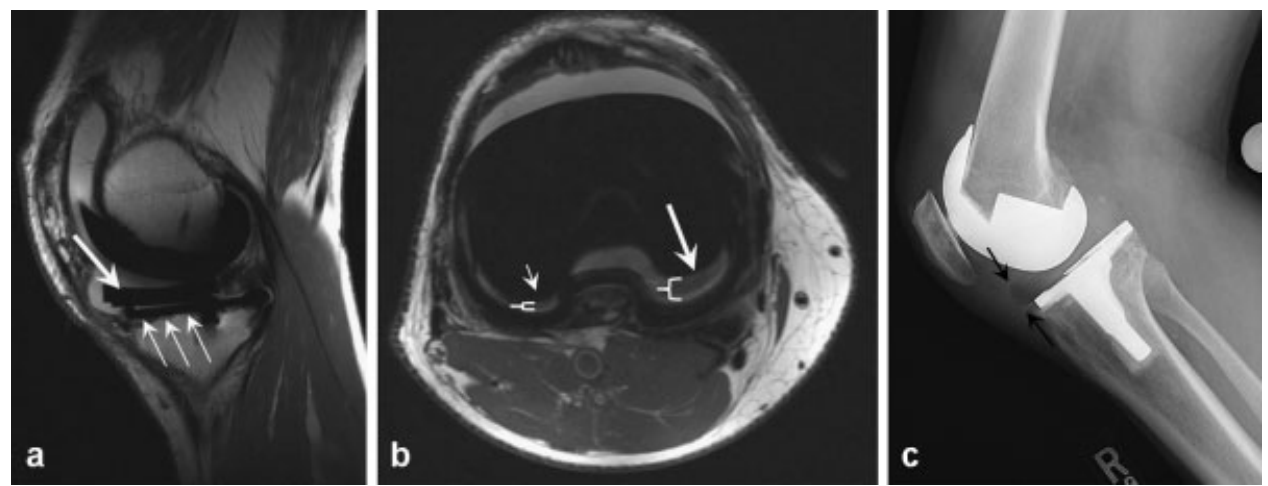

Fig. 6 (a) Sagittal and (b) axial fast spin-echo MR images in a 63-year-old man status post right total knee arthroplasty 16 months ago now with pain, swelling, and clicking. The polyethylene component (thick arrow, a) has separated and is anteriorly displaced relative to the baseplate (thin arrows, a). The axial image (b) demonstrates rotation of the polyethylene component with greater distance (brackets) between its posterior medial (thick long arrow) and lateral (thin short arrow) margins and the baseplate. The anterior displacement of the polyethylene (black arrows) relative to the tibial baseplate can also be appreciated on the lateral radiograph (c).

the surface of the tibial component. ${ }^{25}$ Dissociation of the polyethylene liner from the tibial baseplate is uncommon but has been reported several times in the literature (-Fig. 6). ${ }^{26-30}$ Before the development of specialized pulse sequences, such as the MAVRIC sequence approved by the Food and Drug Administration, metallic artifact from the femoral and tibial components rendered evaluation of the tibial polyethylene insert nearly impossible. The MAVRIC sequence has afforded the opportunity to evaluate for subtle dislodgment and fracture of the polyethylene insert that may have otherwise gone unrecognized (-Fig. 1). The locking pin can also become dislodged, rendering the polyethylene insert potentially unstable. Three-dimensional computer modeling of the polyethylene insert using the sagittal MAVRIC sequence can also be performed with the goal of identifying focal sites of wear and subtle malalignment relative to the tibial baseplate.

\section{Component Malrotation}

In general, TKA is a successful procedure with satisfaction rates $>80 \% .{ }^{31}$ However, in patients with unexplained anterior knee pain, minor rotational malalignment of the TKA components has been implicated as a cause. Excessive rotation, either internal or external, of the femoral component increases femorotibial shear forces and alters normal patellar tracking, and internal rotational errors of the tibial component have also been linked to pain. ${ }^{32-34}$ Revision TKA for pain ascribed to component malrotation has been beneficial in improving functional outcome scores and range of motion. ${ }^{35} \mathrm{CT}$ has traditionally been used to evaluate rotational alignment following total knee replacement. However, MRI is also capable of identifying the anatomical landmarks of the distal femur ${ }^{36}$ necessary for evaluating component position, and it can also determine the signal characteristics and volume of synovitis. Murakami et al demonstrated high interobserver agreement in determining rotational malalignment of knee replacements using MRI and a statistically significant association be- tween the presence and severity of synovitis and pain. ${ }^{37}$ Synovitis related to malrotation typically manifests as homogeneously high signal intensity distention of the capsule.

\section{Extensor Mechanism}

Extensor mechanism injuries following TKA have a reported prevalence of up to $12 \%^{38}$ and can pose unique management challenges for orthopedists. ${ }^{39}$ Injuries include patellar and quadriceps tendon ruptures, soft tissue impingement, and fracture and osteonecrosis of the patella. ${ }^{39}$ In the perioperative setting, a tear along the medial parapatellar arthrotomy site, the most common surgical approach used in TKA, can propagate both superiorly and inferiorly to involve the quadriceps and patellar tendons, respectively (-Fig. 7). Risk
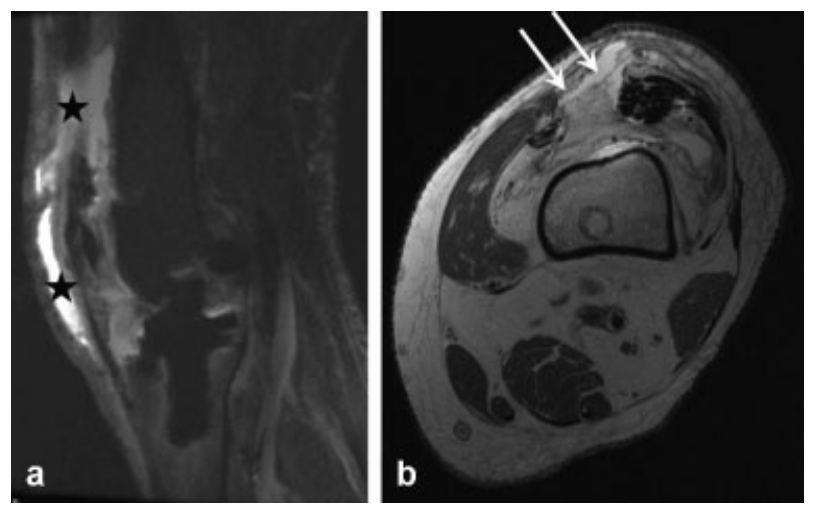

Fig. 7 (a) Sagittal multiacquisition variable resonance image combination inversion recovery and (b) axial fast spin-echo MR images in a 69-year-old man who felt a pop while walking status post right total knee arthroplasty 1 week prior. A vertically oriented defect along the medial margin of the quadriceps tendon (arrows, b) extends along the medial margin of the patellar tendon (not shown here), representing dehiscence at the site of repair of the medial parapatellar surgical incision. There is resultant lateral patellar subluxation and extracapsular fluid extension (stars, a) from the supra- and infrapatellar bursae. 

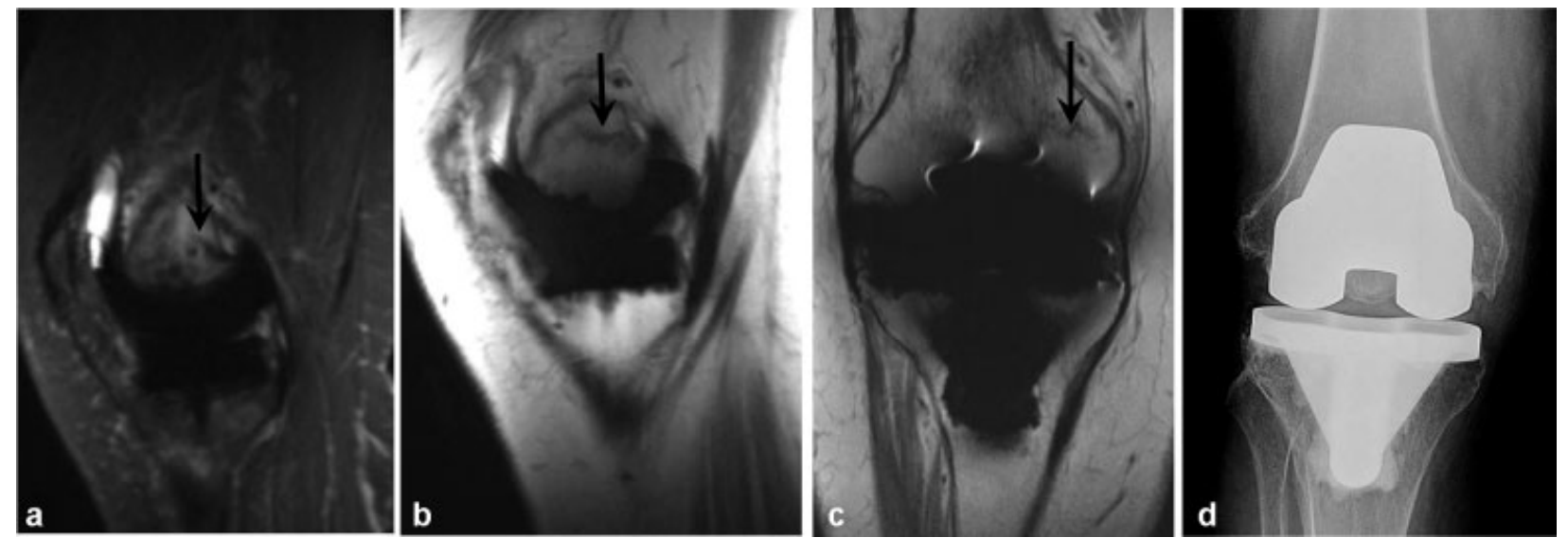

Fig. 8 (a) Sagittal multiacquisition variable-resonance image combination inversion recovery and (b, c) sagittal and coronal fast spin-echo MR images in a 77-year-old woman status post right total knee arthroplasty 15 years prior demonstrate an insufficiency fracture of the medial femoral condyle (arrows) with surrounding marrow edema (a). (d) The fracture line cannot be identified on the corresponding radiograph.

factors for periprosthetic patellar fracture include patellar resurfacing, especially with excessive resection, use of a patellar implant with a large central plug, use of a cementless implant, and lateral release. ${ }^{40}$ Radiographs and CT are both insensitive to extensor mechanism injuries, and radiographs may fail to identify a patellar fracture unless displaced. Combined short tau inversion recovery and MAVRIC pulse sequence techniques MAVRIC IR can detect bone marrow and soft tissue edema, even when directly adjacent to the implant (-Fig. 8). Surgical technique during TKA placement may result in disruption of the anastomotic vascular ring around the patella and resultant osteonecrosis. Although lateral and Merchant radiographic views may depict sclerosis, flattening, and fragmentation of bone in advanced cases, MRI may make the diagnosis earlier. $^{39}$

\section{Patellar Clunk Syndrome}

Patellar clunk syndrome is a painful condition resulting from mechanical catching or "clunking" during active extension following TKA ${ }^{41}$ and was originally described by Hozack et al who discovered a prominent fibrous nodule at the junction of the proximal patellar pole and quadriceps tendon in a posterior stabilized (cruciate-substituting) TKA. $^{42}$ The nodule is thought to represent postsurgical scar formation related to soft tissue impingement by the sharp anterosuperior edge of the TKA at the intercondylar notch. ${ }^{43}$ In knee flexion, the nodule becomes entrapped within the notch as the quadriceps tendon and patella migrate cephalad. Then, as the knee is being extended, at 30 to 45 degrees from full extension, the fibrous nodule painfully and sometimes audibly "clunks" out of the intercondylar notch. ${ }^{20}$ The diagnosis is regularly made on clinical examination, but it is important to distinguish this entity from other sources of patellofemoral pain. MRI can confirm the diagnosis by reliably identifying and reproducibly providing size dimensions of the soft tissue nodule and decision support for arthroscopic debridement in symptomatic patients ( - Fig. 9). ${ }^{41}$ Ultrasound may also be bene- ficial by confirming the movement of the nodule during knee flexion and extension in real time while also providing size dimensions of the nodule. ${ }^{44}$

\section{Vascular Complications}

A less common complication related to TKA is recurrent hemarthrosis, but when it occurs it may be disabling by severely restricting motion and inducing painful swelling. ${ }^{45}$ Although poorly understood, it may result from arthroplasty components impinging on hypervascular

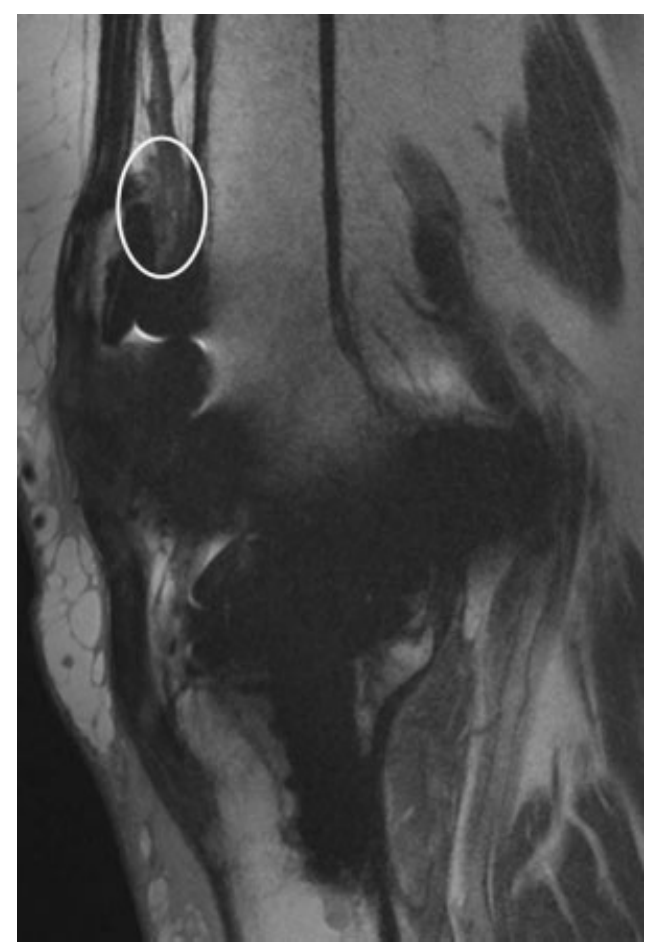

Fig. 9 Sagittal fast spin-echo MR image in a 58-year-old woman status post right total knee arthroplasty 1 year ago now with anterior knee pain demonstrates a focus of scarred synovium (oval) along the posterosuperior margin of the patella that may be implicated in patellar clunk syndrome. 

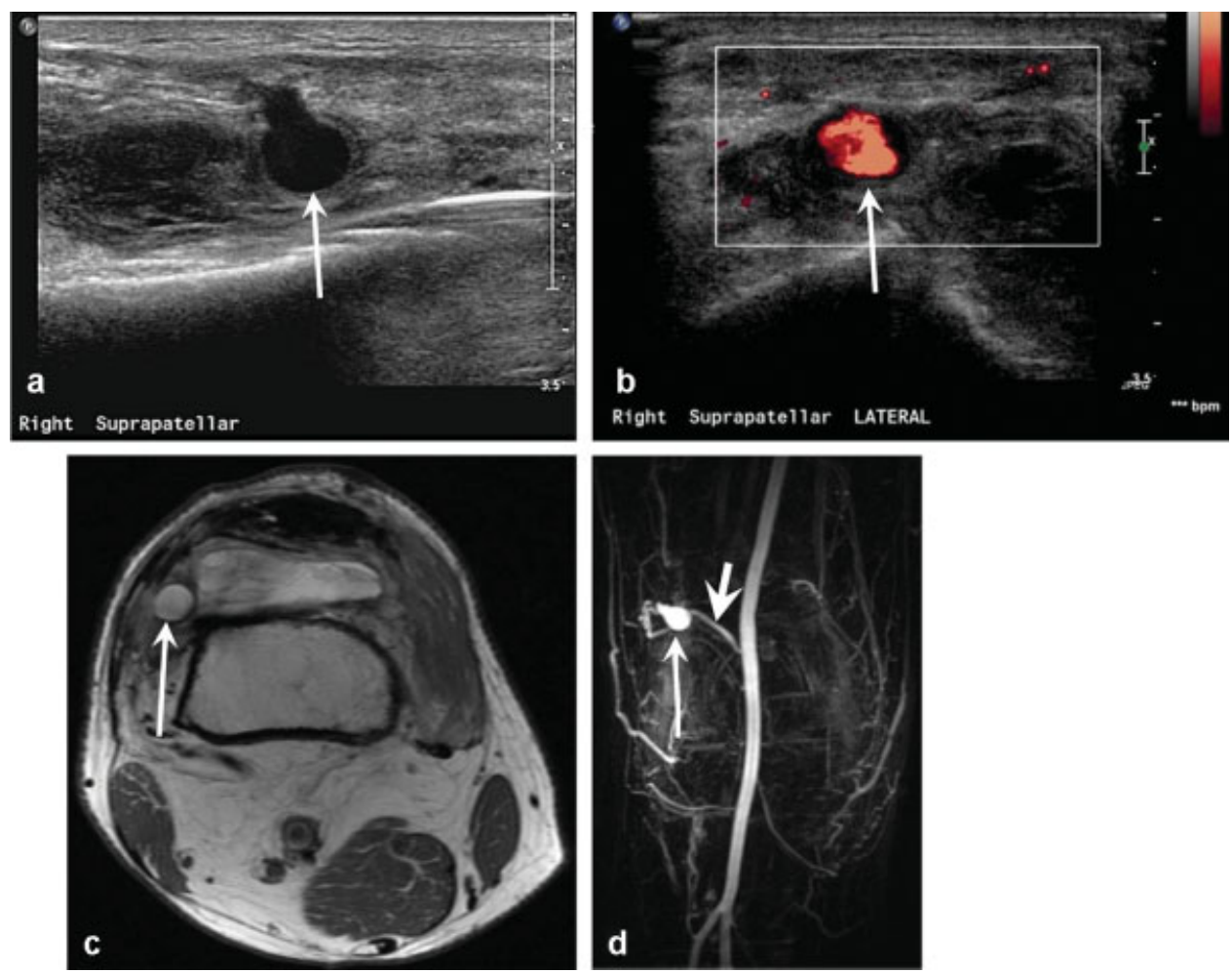

Fig. 10 A 62-year-old man status post right total knee arthroplasty 2 years prior and synovectomy for infection 3 months prior with recent hemarthrosis on joint aspiration. Ultrasound of the right knee (a) with and (b) without power Doppler demonstrates a prominent vascular structure (arrows) within the lateral suprapatellar recess. (c) Axial fast spin-echo MR image and (d) coronal maximal intensity projection image from a time intensity sensitive contrast-enhanced magnetic resonance angiography examination confirms the suspected pseudoaneurysm (long thin arrows) arising from the superior lateral geniculate artery (short thick arrow).

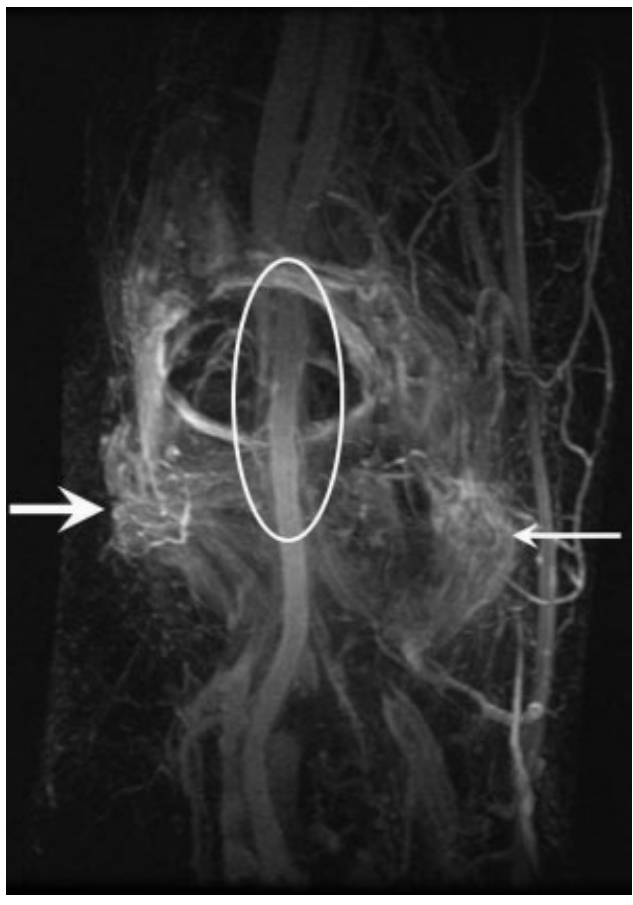

Fig. 11 Coronal maximal intensity projection image from a time intensity sensitive contrast-enhanced magnetic resonance angiography examination in a 77-year-old man with recurrent hemarthrosis status post right total knee arthroplasty 18 months prior demonstrates synovial hypervascularity most prominent within the inferomedial (long thin arrow) and inferolateral (small thick arrow) geniculate distributions, but without a dominant supplying geniculate artery identified. Incidental note is made of mild contour irregularity of the mid-popliteal artery (oval) reflecting atherosclerotic disease. proliferative synovium. ${ }^{46}$ Other causes include pseudoaneurysms (-Fig. 10), arteriovenous fistulae, pigmented villonodular synovitis, and component loosening resulting in micromotion and bleeding limb elevation and rest of the adjacent bone. Conservative management includes joint aspiration and cryotherapy. If conservative treatment fails, open or arthroscopic synovectomy or conventional angiography with transcatheter embolization can be performed. Digital subtraction contrast-enhanced magnetic resonance angiography (MRA) can offer valuable information before definitive treatment, by providing a road map of the vascular anatomy and identify a dominant artery or arteries supplying the hypervascular synovium that can be targeted for subsequent embolization or synovectomy (-Fig. 11). ${ }^{46}$

\section{Conclusion}

Optimization of existing pulse sequences and advent of newer specialized pulse sequences to reduce metallic artifact have enhanced enabled MRIs capability in evaluating complications related to TKA, particularly in the setting of nonspecific knee pain. Given the variety of arthroplasty designs, a knowledge of the polyethylene insert may be helpful in discerning subtle areas of wear and detachment. With its superior soft tissue contrast, MRI can differentiate between synovial patterns and in particular, polymeric-induced verses septic synovitis from infection. 


\section{References}

1 Healthcare Cost and Utilization Project (HCUP). Nationwide Inpatient Sample (NIS). Agency for Healthcare Research and Quality; 1999-2009. http://hcupnet.ahrq.gov/. Accessed August 24, 2014

2 Losina E, Thornhill TS, Rome BN, Wright J, Katz JN. The dramatic increase in total knee replacement utilization rates in the United States cannot be fully explained by growth in population size and the obesity epidemic. J Bone Joint Surg Am 2012;94(3): 201-207

3 Cram P, Lu X, Kates SL, Singh JA, Li Y, Wolf BR. Total knee arthroplasty volume, utilization, and outcomes among Medicare beneficiaries, 1991-2010. JAMA 2012;308(12):1227-1236

4 Solomon LB, Stamenkov RB, MacDonald AJ, et al. Imaging periprosthetic osteolysis around total knee arthroplasties using a human cadaver model. J Arthroplasty 2012;27(6):1069-1074

5 Reish TG, Clarke HD, Scuderi GR, Math KR, Scott WN. Use of multidetector computed tomography for the detection of periprosthetic osteolysis in total knee arthroplasty. J Knee Surg 2006;19(4): 259-264

6 Subhas N, Primak AN, Obuchowski NA, et al. Iterative metal artifact reduction: Evaluation and optimization of technique. Skeletal Radiol 2014;43(12):1729-1735

7 Koch KM, Lorbiecki JE, Hinks RS, King KF. A multispectral threedimensional acquisition technique for imaging near metal implants. Magn Reson Med 2009;61(2):381-390

8 Lu W, Pauly KB, Gold GE, Pauly JM, Hargreaves BA. SEMAC: slice encoding for metal artifact correction in MRI. Magn Reson Med 2009;62(1):66-76

9 Chen CA, Chen W, Goodman SB, et al. New MR imaging methods for metallic implants in the knee: artifact correction and clinical impact. J Magn Reson Imaging 2011;33(5):1121-1127

10 Sharkey PF, Lichstein PM, Shen C, Tokarski AT, Parvizi J. Why are total knee arthroplasties failing today-has anything changed after 10 years? J Arthroplasty 2014;29(9):1774-1778

11 Mulcahy H, Chew FS. Current concepts in knee replacement: features and imaging assessment. AJR Am J Roentgenol 2013; 201(6):W828-W842

12 Fritz J, Lurie B, Miller TT, Potter HG. MR imaging of hip arthroplasty implants. Radiographics 2014;34(4):E106-E132

13 Bosetti M, Massè A, Navone R, Cannas M. Biochemical and histological evaluation of human synovial-like membrane around failed total hip replacement prostheses during in vitro mechanical loading. J Mater Sci Mater Med 2001;12(8):693-698

14 Nam D, Bostrom MP, Fahlgren A. Emerging ideas: Instabilityinduced periprosthetic osteolysis is not dependent on the fibrous tissue interface. Clin Orthop Relat Res 2013;471(6):1758-1762

15 Bauer TW, Schils J. The pathology of total joint arthroplasty. II. Mechanisms of implant failure. Skeletal Radiol 1999;28(9): 483-497

16 Howie DW, Neale SD, Martin W, et al. Progression of periacetabular osteolytic lesions. J Bone Joint Surg Am 2012;94(16): e1171-e1176

17 Heyse TJ, Chong R, Davis J, Boettner F, Haas SB, Potter HG. MRI analysis of the component-bone interface after TKA. Knee 2012; 19(4):290-294

18 Hayter CL, Koff MF, Shah P, Koch KM, Miller TT, Potter HG. MRI after arthroplasty: comparison of MAVRIC and conventional fast spin-echo techniques. AJR Am J Roentgenol 2011;197(3): W405-W411

19 Lachiewicz PF, Geyer MR. The use of highly cross-linked polyethylene in total knee arthroplasty. J Am Acad Orthop Surg 2011; 19(3):143-151

20 Mulcahy H, Chew FS. Current concepts in knee replacement: complications. AJR Am J Roentgenol 2014;202(1):W76-W86

21 Ries MD, Pruitt L. Effect of cross-linking on the microstructure and mechanical properties of ultra-high molecular weight polyethylene. Clin Orthop Relat Res 2005;440(440):149-156
22 Garvin KL, Konigsberg BS. Infection following total knee arthroplasty: prevention and management. J Bone Joint Surg Am 2011; 93(12):1167-1175

23 Love C, Tomas MB, Tronco GG, Palestro CJ. FDG PET of infection and inflammation. Radiographics 2005;25(5):1357-1368

24 Plodkowski AJ, Hayter CL, Miller TT, Nguyen JT, Potter HG. Lamellated hyperintense synovitis: potential MR imaging sign of an infected knee arthroplasty. Radiology 2013;266(1):256-260

25 Huang CH, Liau JJ, Cheng CK. Fixed or mobile-bearing total knee arthroplasty. J Orthop Surg 2007;2:1

26 Anderson JAA, MacDessi SJ, Della Valle AG. Spontaneous, recurrent dislodgment of the polyethylene tibial insert after total knee arthroplasty. A case report. J Bone Joint Surg Am 2007;89(2):404-407

27 Ries MD. Dissociation of an ultra-high molecular weight polyethylene insert from the tibial baseplate after total knee arthroplasty. A case report. J Bone Joint Surg Am 2004;86-A(7):1522-1524

28 Davis PF, Bocell JR Jr, Tullos HS. Dissociation of the tibial component in total knee replacements. Clin Orthop Relat Res 1991;(272): 199-204

29 Hedlundh U, Andersson M, Enskog L, Gedin P. Traumatic late dissociation of the polyethylene articulating surface in a total knee arthroplasty-a case report. Acta Orthop Scand 2000;71(5): 532-533

30 Poulter RJ, Ashworth MJ. A case of dissociation of polyethylene from its metal baseplate in a "one piece" compression-moulded AGC tibial component. Knee 2005;12(3):243-244

31 Robertsson O, Dunbar M, Pehrsson T, Knutson K, Lidgren L. Patient satisfaction after knee arthroplasty: a report on 27,372 knees operated on between 1981 and 1995 in Sweden. Acta Orthop Scand 2000;71(3):262-267

32 Barrack RL, Schrader T, Bertot AJ, Wolfe MW, Myers L. Component rotation and anterior knee pain after total knee arthroplasty. Clin Orthop Relat Res 2001;(392):46-55

33 Nicoll D, Rowley DI. Internal rotational error of the tibial component is a major cause of pain after total knee replacement. J Bone Joint Surg Br 2010;92(9):1238-1244

34 Cameron HU. A comedy of errors: the bad knee. J Arthroplasty 2005;20(4, Suppl 2):18-22

35 Lakstein D, Zarrabian M, Kosashvili Y, Safir O, Gross AE, Backstein D. Revision total knee arthroplasty for component malrotation is highly beneficial: a case control study. J Arthroplasty 2010;25(7): 1047-1052

36 Griffin FM, Math K, Scuderi GR, Insall JN, Poilvache PL. Anatomy of the epicondyles of the distal femur: MRI analysis of normal knees. J Arthroplasty 2000;15(3):354-359

37 Murakami AM, Hash TW, Hepinstall MS, Lyman S, Nestor BJ, Potter HG. MRI evaluation of rotational alignment and synovitis in patients with pain after total knee replacement. J Bone Joint Surg Br 2012;94(9):1209-1215

38 Parker DA, Dunbar MJ, Rorabeck CH. Extensor mechanism failure associated with total knee arthroplasty: prevention and management. J Am Acad Orthop Surg 2003;11(4):238-247

39 Nam D, Abdel MP, Cross MB, et al. The management of extensor mechanism complications in total knee arthroplasty. AAOS exhibit selection. J Bone Joint Surg Am 2014;96(6):e47

40 Chalidis BE, Tsiridis E, Tragas AA, Stavrou Z, Giannoudis PV. Management of periprosthetic patellar fractures. A systematic review of literature. Injury 2007;38(6):714-724

41 Heyse TJ, Chong R, Davis J, Haas SB, Figgie MP, Potter HG. MRI diagnosis of patellar clunk syndrome following total knee arthroplasty. HSS J 2012;8(2):92-95

42 Hozack WJ, Rothman RH, Booth RE Jr, Balderston RA. The patellar clunk syndrome. A complication of posterior stabilized total knee arthroplasty. Clin Orthop Relat Res 1989;(241):203-208

43 Beight JL, Yao B, Hozack WJ, Hearn SL, Booth RE Jr. The patellar "clunk" syndrome after posterior stabilized total knee arthroplasty. Clin Orthop Relat Res 1994;(299):139-142 
48 MRI Evaluation of the Painful Total Knee Arthroplasty Sneag et al.

44 Okamoto T, Futani H, Atsui K, Fukunishi S, Koezuka A, Maruo S. Sonographic appearance of fibrous nodules in patellar clunk syndrome: a case report. J Orthop Sci 2002;7(5):590-593

45 Rukavina A, Kerkhoffs GM, Schneider P, Kuster MS. Recurrent hemarthrosis after total knee arthroplasty. Knee Surg Sports Traumatol Arthrosc 2010;18(7):898-900
46 Hash TW II, Maderazo AB, Haas SB, Saboeiro GR, Trost DW, Potter HG. Magnetic resonance angiography in the management of recurrent hemarthrosis after total knee arthroplasty. J Arthroplasty 2011;26(8):1357-61.e1 